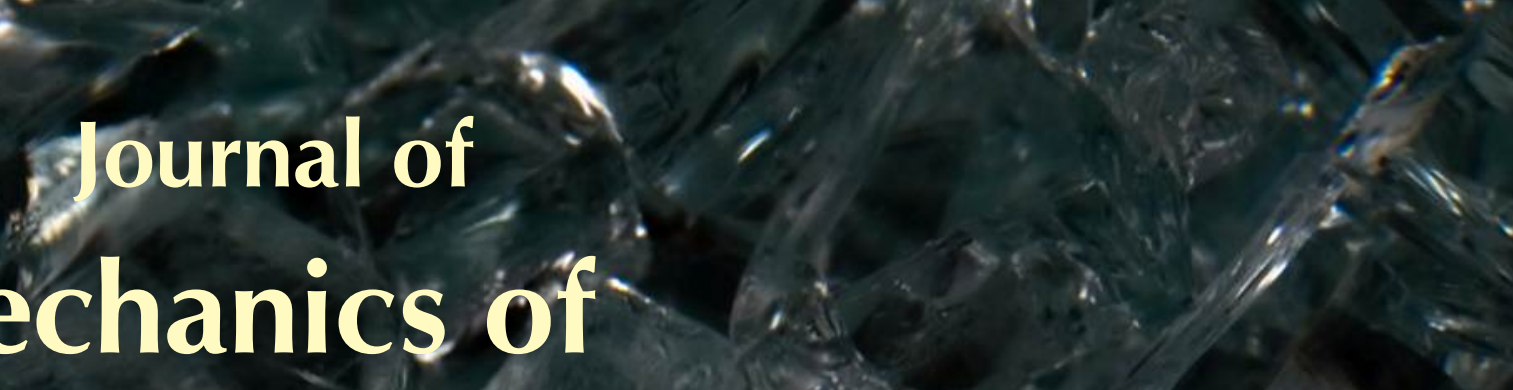

Materials and Structures

Mechanics of

0.

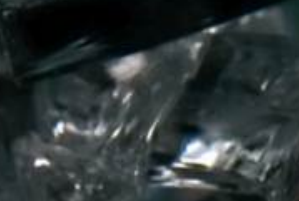

$+8$

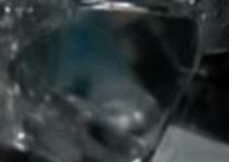

$y=1$

B⿺

I \& GREEN'S FUNCTION FOR SYMMETRIC LOADING OF" AN ELASTIC SPIIERE WITH APPLICATION TO CONTACT PROBLEMS Alexey S. Titovich and Andrew N. Norris
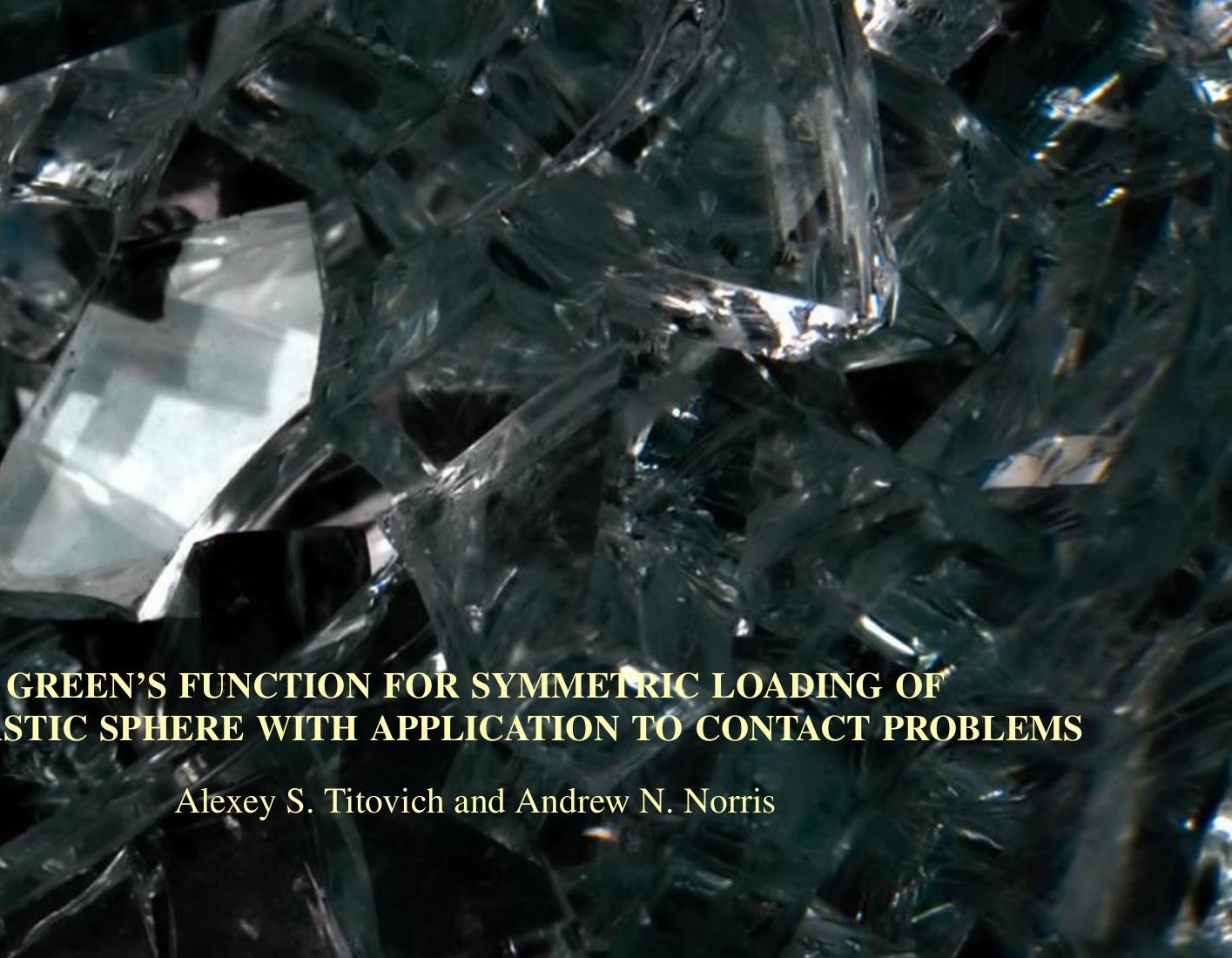

1.

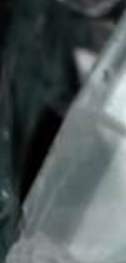




\title{
GREEN'S FUNCTION FOR SYMMETRIC LOADING OF AN ELASTIC SPHERE WITH APPLICATION TO CONTACT PROBLEMS
}

\author{
Alexey S. Titovich AND Andrew N. NorRis
}

\begin{abstract}
A compact form for the static Green's function for symmetric loading of an elastic sphere is derived. The expression captures the singularity in closed form using standard functions and quickly convergent series. Applications to problems involving contact between elastic spheres are discussed. An exact solution for a point load on a sphere is presented and subsequently generalized for distributed loads. Examples for constant and Hertzian-type distributed loads are provided, where the latter is also compared to the Hertz contact theory for identical spheres. The results show that the form of the loading assumed in Hertz contact theory is valid for contact angles up to about ten degrees. For larger angles, the actual displacement is smaller and the contact surface is no longer flat.
\end{abstract}

\section{Introduction}

Contact between spheres has intrigued researchers for more than a century, and still no simple closedform analytical solution exists. One of the first and most important developments in the field, due to Heinrich Hertz [1881], is an approximate solution for the normal, frictionless contact of linear elastic spheres. The major assumption in Hertz's model was that the contact area is small compared to the radii of curvature, which has served as a useful engineering approximation in many applications. Ever since then many have tried to relax this assumption while maintaining a compact, workable solution. The Green's function for symmetric loading on a sphere provides the means to find the exact response for arbitrary loading, a first step towards improving on Hertz's classic solution. Existing forms of the Green's function are however not suitable for fast and ready computation, either due to slow convergence of series or analytically cumbersome expressions. The goal of the present paper is to provide an alternative form of the Green's function suitable for fast computation of solutions under arbitrary loading.

Sternberg and Rosenthal [1952] present an in-depth study of the nature of the singularities on an elastic sphere loaded by two opposing concentrated point forces. As expected, the dominant inverse square singularity in the stress components can be removed by subtraction of an appropriate multiple of Boussinesq's solution for a point load at the surface of a half space. Sternberg and Rosenthal showed that the quickly convergent residual field retains a weaker singularity of logarithmic form, a result that is also evident in the solution developed here. The singular solutions obtained by Sternberg and Rosenthal were extended to arbitrarily oriented point forces in [Guerrero and Turteltaub 1972]. Our interest here is in developing an analogous separation of the Green's function (circular ring loading). In this regard, a relatively compact form of the Green's function for the sphere was derived by Bondareva [1969] who used it to solve the problem of the weighted sphere. In [Bondareva 1971], she formulates an example with a sphere contacting a rigid surface. This has been used to solve for the rebound of a sphere from a

Keywords: Green's function, sphere, contact. 
surface [Villaggio 1996]. Bondareva's solution starts with the known series expansion [Lur'e 1955] for the solution of the elasticity problem of a sphere, and replaces it with finite integrals of known functions.

In this paper we introduce an alternative form for the Green's function for a sphere, comprised of analytical functions and a quickly convergent series. No direct integration is required. The methodology for determining the analytical functions is motivated by the simple example of a point load on a sphere, for which we derive a solution similar in spirit to that of [Sternberg and Rosenthal 1952], but using a fundamentally different approach: partial summation of infinite series as compared with a functional ansatz. The present methods allows us to readily generalize the point-load solution to arbitrary symmetric normal loading. A typical contact problem involves solving a complicated integral equation for the contact stress once a displacement is specified. Instead, we will use the derived Green's function in the direct sense, solving for the displacements for a given load. This is used to check the validity of Hertz contact theory through the assumed form of the stress distribution.

The outline of the paper is as follows. The known series solution for symmetric loading on a sphere is reviewed in Section 2. The proposed method for simplification is first illustrated in Section 3 by deriving a quickly convergent form of the solution for a point force. The Green's function for symmetric loading is then developed in Section 4, and is illustrated by application to different loadings. Conclusions are given in Section 5.

\section{Series solution}

Consider a solid sphere of radius $R$, with surface $r=R, 0 \leq \theta \leq \pi$, in spherical polar coordinates $(r, \theta, \phi)$. The sphere is linear elastic with shear modulus $G$ and Poisson's ratio $v$. The surface is subject to tractions

$$
\sigma_{r \theta}=0, \quad \sigma_{r \phi}=0, \quad \sigma_{r r}=\sigma(\theta) \quad \text { for } r=R, 0 \leq \theta \leq \pi, 0 \leq \phi<2 \pi .
$$

Using the known properties of Legendre functions, see (45), allows us to express the normal stress as

$$
\sigma(\theta)=\frac{1}{2} \sum_{n=0}^{\infty}(2 n+1) \sigma_{n} P_{n}(\cos \theta),
$$

where the Legendre series coefficients are

$$
\sigma_{n}=\int_{0}^{\pi} \sigma(\phi) P_{n}(\cos \phi) \sin \phi d \phi .
$$

The displacements and tractions for the sphere can also be represented in series form [Zhupanska 2011, Equation (5)]:

$$
\begin{aligned}
& 2 G u_{r}=\sum_{n=0}^{\infty}\left[(n-2+4 v) A_{n} r+B_{n} r^{-1}\right] r^{n} P_{n}(\cos \theta), \\
& 2 G u_{\theta}=\sum_{n=1}^{\infty}\left[n(n+5-4 v) A_{n} r+(n+1) B_{n} r^{-1}\right] r^{n} \frac{P_{n}^{1}(\cos \theta)}{n(n+1)},
\end{aligned}
$$




$$
\begin{aligned}
\sigma_{r r} & =\sum_{n=0}^{\infty}\left[[n(n-1)-2(1+v)] A_{n}+(n-1) B_{n} r^{-2}\right] r^{n} P_{n}(\cos \theta), \\
\sigma_{r \theta} & =\sum_{n=1}^{\infty}\left[n[(n-1)(n+3)+2(1+v)] A_{n}+\left(n^{2}-1\right) B_{n} r^{-2}\right] r^{n} \frac{P_{n}^{1}(\cos \theta)}{n(n+1)},
\end{aligned}
$$

with $B_{0} \equiv 0$, and where $B_{1}$ corresponds to a rigid body translation via $2 G \boldsymbol{u}(0, \cdot)=B_{1} \boldsymbol{e}_{z}$. It follows from (1) that

$$
\begin{aligned}
A_{0} & =\frac{-\sigma_{0}}{2(1+v)}, \quad A_{1}=0, \\
A_{n} & =-\frac{\sigma_{n}}{4 R^{n}} \frac{(n+1)(2 n+1)}{[n(n-1)+(2 n+1)(1+v)]}, \quad n \geq 2, \\
B_{n} & =\frac{-n}{n^{2}-1}[(n-1)(n+3)+2(1+v)] R^{2} A_{n}, \quad n \geq 2 .
\end{aligned}
$$

Thus, noting that $P_{n}^{1}(\cos \theta)=\frac{d}{d \theta} P_{n}(\cos \theta)$, we have

$$
\begin{aligned}
& u_{r}(R, \theta)=\frac{R}{4 G}\left(\frac{2(1-2 v)}{1+v} \sigma_{0}+\sum_{n=2}^{\infty} \sigma_{n}\left(\frac{2 n+1}{n-1}\right)\left(\frac{2(1-v) n^{2}+v n-1+2 v}{n^{2}+(1+2 v) n+1+v}\right) P_{n}(\cos \theta)\right), \\
& u_{\theta}(R, \theta)=\frac{R}{4 G} \frac{d}{d \theta} \sum_{n=2}^{\infty} \sigma_{n}\left(\frac{2 n+1}{n-1}\right)\left(\frac{(-1+2 v) n+2-v}{n^{2}+(1+2 v) n+1+v}\right) P_{n}(\cos \theta) .
\end{aligned}
$$

Bondareva [1969], using a different representation, replaced the infinite summation of Legendre functions by a combination of closed form expressions and an integral, each dependent on $v$. The integral term contains a logarithmic singularity which, together with the complex-valued nature of its coefficients, makes its evaluation indirect. Here we propose an alternative form for the Green's function in a combination of closed-form expressions and a standard summation of Legendre functions that is, by design, quickly convergent.

\section{Point force}

3.1. Exact solution. In order to illustrate the method, we first consider the simpler problem of the point force of magnitude $F$ applied at $\theta=0$ defined by

$$
\sigma(\theta)=\frac{-F}{2 \pi R^{2}} \lim _{\psi \downarrow 0} \frac{\delta(\theta-\psi)}{\sin \psi} \Longleftrightarrow \sigma_{n}=\frac{-F}{2 \pi R^{2}},
$$

where we have used the property $P_{n}(1)=1$. The difficulty with the infinite summations (6) is twofold: first, this is not a suitable form to reproduce the singular nature of the Green's function; secondly, they do not converge quickly as a function of the truncated value for $n$. The idea here is to replace the summation by closed form expressions plus a summation that is both regular and quickly convergent. 
The fundamental idea behind the present method is to write $u_{r}$ and $u_{\theta}$ of (6) in the form

$$
\begin{aligned}
& u_{r}(R, \theta)=\frac{-F}{8 \pi G R}\left(4(1-v) S(\theta)+\sum_{j=0}^{M} a_{j}(v) S_{j}(\theta)+f(\theta)\right), \\
& u_{\theta}(R, \theta)=\frac{-F}{8 \pi G R} \frac{d}{d \theta}\left(\sum_{j=0}^{M} b_{j}(v) S_{j}(\theta)+g(\theta)\right),
\end{aligned}
$$

where the functions $S(\theta)$ and $S_{j}(\theta)(j=1, \ldots M)$, are closed-form expressions, in this case:

$$
\begin{aligned}
S(\theta) & =\sum_{n=0}^{\infty} P_{n}(\cos \theta)=\frac{1}{2} \csc \frac{\theta}{2}, \\
S_{j}(\theta) & =\sum_{n=0}^{\infty} \frac{P_{n+j}(\cos \theta)}{n+1}, \quad j=0,1, \ldots,
\end{aligned}
$$

and $f(\theta), g(\theta)$ are regular functions of $\theta$ defined by quickly convergent series in $n$,

$$
f(\theta)=\sum_{n=0}^{\infty} C_{n} P_{n}(\cos \theta), \quad g(\theta)=\sum_{n=0}^{\infty} D_{n} P_{n}(\cos \theta) .
$$

The coefficients $a_{0}, a_{1}, \ldots, a_{M}$ are defined so that $C_{n}=\mathrm{O}\left(n^{-(M+2)}\right)$ as $n \rightarrow \infty$. This criterion uniquely provides the constants $a_{0}, a_{1}, \ldots, a_{M}$ as solutions of a system of linear equations. Similarly, $b_{0}, b_{1}, \ldots$, $b_{M}$ are uniquely defined by $D_{n}=\mathrm{O}\left(n^{-(M+2)}\right)$ as $n \rightarrow \infty$.

Here we consider the specific case of $M=2$. Other values of $M$ could be treated in the same manner; however, we will show that $M=2$ is adequate for the purpose of improving convergence. In this case (8) becomes

$$
\begin{aligned}
\begin{aligned}
u_{r}(R, \theta)= & \frac{-F}{8 \pi G R}\left[4(1-v) S(\theta)+a_{0} S_{0}(\theta)+a_{1} S_{1}(\theta)+a_{2} S_{2}(\theta)+f(\theta)\right] \\
= & \frac{-F}{8 \pi G R}\left[\sum_{n=2}^{\infty}\left(4(1-v)+\frac{a_{0}}{n+1}+\frac{a_{1}}{n}+\frac{a_{2}}{n-1}+C_{n}\right) P_{n}(\theta)+C_{0} P_{0}(\theta)+C_{1} P_{1}(\theta)\right. \\
& \left.\quad+4(1-v)\left(P_{0}(\theta)+P_{1}(\theta)\right)+a_{0}\left(P_{0}(\theta)+\frac{1}{2} P_{1}(\theta)\right)+a_{1} P_{1}(\theta)\right], \\
u_{\theta}(R, \theta)= & \frac{-F}{8 \pi G R} \frac{d}{d \theta}\left[b_{0} S_{0}(\theta)+b_{1} S_{1}(\theta)+b_{2} S_{2}(\theta)+g(\theta)\right] \\
= & \frac{-F}{8 \pi G R} \frac{d}{d \theta}\left[\sum_{n=2}^{\infty}\left(\frac{b_{0}}{n+1}+\frac{b_{1}}{n}+\frac{b_{2}}{n-1}+D_{n}\right) P_{n}(\theta)\right. \\
& \left.\quad+b_{0}\left(P_{0}(\theta)+\frac{1}{2} P_{1}(\theta)\right)+b_{1} P_{1}(\theta)+D_{0} P_{0}(\theta)+D_{1} P_{1}(\theta)\right],
\end{aligned}
\end{aligned}
$$

where the associated functions $S_{j}(\theta), j=0,1,2$, are (see Appendix B) 


$$
\begin{aligned}
& S_{0}(\theta)=\log \left(1+\csc \frac{\theta}{2}\right), \\
& S_{1}(\theta)=-S_{0}(\theta)-2 \log \sin \frac{\theta}{2}, \\
& S_{2}(\theta)=S_{1}(\theta) \cos \theta-2 \sin \frac{\theta}{2}\left(1-\sin \frac{\theta}{2}\right) .
\end{aligned}
$$

Equations (8), (9a), and (12) indicate the expected Boussinesq-like $\theta^{-1}$ singularity as well as the weaker $\log \theta$ singularity first described in [Sternberg and Rosenthal 1952]. The logarithmic singularities in $S_{j}(\theta), j=0,1,2$, can be compared to the potential functions [ $\left.D_{1}\right],\left[D_{2}\right]$, and [ $\left.D_{3}\right]$ in Equation (17) of that reference, which provide a logarithmic singularity. In the present notation these are, respectively (using capital $\Phi$ so as not to be confused with the angle $\phi$, and making the substitution $\theta \rightarrow \pi-\theta$ ),

$$
\begin{aligned}
& \Phi_{1}(\theta)=2 \log \sin \frac{\theta}{2}, \quad \Phi_{2}(\theta)=-R\left(1+2 \cos \theta \log \sin \frac{\theta}{2}\right) \\
& \Phi_{3}(\theta)=R^{2}\left(2\left(1-3 \cos ^{2} \theta\right) \log \sin \frac{\theta}{2}+\cos ^{2} \theta-3 \cos \theta-1\right) .
\end{aligned}
$$

These clearly display the same form of the singularity as in equations (12), but are otherwise different.

Define the first two coefficients of $f(\theta)$ and $g(\theta)$ from (10) as

$$
\begin{array}{ll}
C_{0}=\frac{2(1-2 v)}{1+v}-4(1-v)-a_{0}, & C_{1}=-4(1-v)-\frac{1}{2} a_{0}-a_{1}, \\
D_{0}=-b_{0}, & D_{1}=-\frac{1}{2} b_{0}-b_{1} .
\end{array}
$$

The coefficients $a_{n}$ and $b_{n}$ are then found by comparing expression (11) to the series solution in (6), expanding both expressions for large $n$, and equating the coefficients of the same order terms. Thus, the original assumed form of the solution (8) implies

$$
\begin{aligned}
\sum_{n=2}^{\infty} \frac{P_{n}(\theta)}{n^{2}+(1+2 v) n+1+v}\left(\frac{2 n+1}{n-1}\right) \times\left\{\begin{array}{l}
\left(2(1-v) n^{2}+v n-1+2 v\right) \\
((-1+2 v) n+2-v)
\end{array}\right. \\
=\sum_{n=2}^{\infty} P_{n}(\theta) \times\left\{\begin{array}{l}
\left(4(1-v)+\frac{a_{0}}{n+1}+\frac{a_{1}}{n}+\frac{a_{2}}{n-1}+C_{n}\right) \\
\left(\frac{b_{0}}{n+1}+\frac{b_{1}}{n}+\frac{b_{2}}{n-1}+D_{n}\right)
\end{array}\right.
\end{aligned}
$$

where

$$
\begin{aligned}
& a_{0}=\frac{1}{2}(1+v)(1-2 v)\left(-16 v^{2}+8 v+5\right), \\
& a_{1}=-32 v^{4}+16 v^{3}+30 v^{2}-16 v-1, \\
& a_{2}=16 v^{4}-16 v^{3}-5 v^{2}+\frac{13}{2} v+\frac{1}{2}, \\
& b_{0}=\frac{1}{2}(1+v)\left(16 v^{2}-12 v-1\right), \\
& b_{1}=-16 v^{3}+4 v^{2}+13 v-4, \\
& b_{2}=8 v^{3}-6 v^{2}-\frac{5}{2} v+\frac{5}{2}
\end{aligned}
$$


The remaining coefficients $C_{n}$ and $D_{n}$ are then determined directly from (15):

$$
\begin{aligned}
C_{n} & =\frac{(1+v)}{L_{n}}\left(\left(6-a_{1}-6 a_{2}\right) n+a_{1}\right) \\
& =-\frac{(1+v)}{L_{n}}\left(\left(64 v^{4}-80 v^{3}+23 v-4\right) n+\left(32 v^{4}-16 v^{3}-30 v^{2}+16 v+1\right)\right), \\
D_{n} & =\frac{(1+v)}{L_{n}}\left(\left(6-b_{1}-6 b_{2}\right) n+b_{1}\right) \\
& =-\frac{(1+v)}{L_{n}}\left(\left(32 v^{3}-32 v^{2}-2 v+5\right) n+\left(16 v^{3}-4 v^{2}-13 v+4\right)\right),
\end{aligned}
$$

where

$$
L_{n} \equiv n\left(n^{2}-1\right)\left(n^{2}+(1+2 v) n+1+v\right) .
$$

In summary, the new form of the point force solution is given by the displacements in (11) where the functions and coefficients are given in (12)-(14) and (16)-(18).

3.2. Numerical examples. In the following examples we introduce the integer $N$ as the truncation value of the series in (10). The Poisson's ratio was taken to be 0.4. Displacements have been normalized by the constant coefficient of the series as $U_{i}=-8 \pi G R F^{-1} u_{i}$, where $i=r, \theta$. Figure 1 shows the rate of convergence of the displacements given by (11), whereas Figures 2 and 3 compare the displacements in (6) with (11).
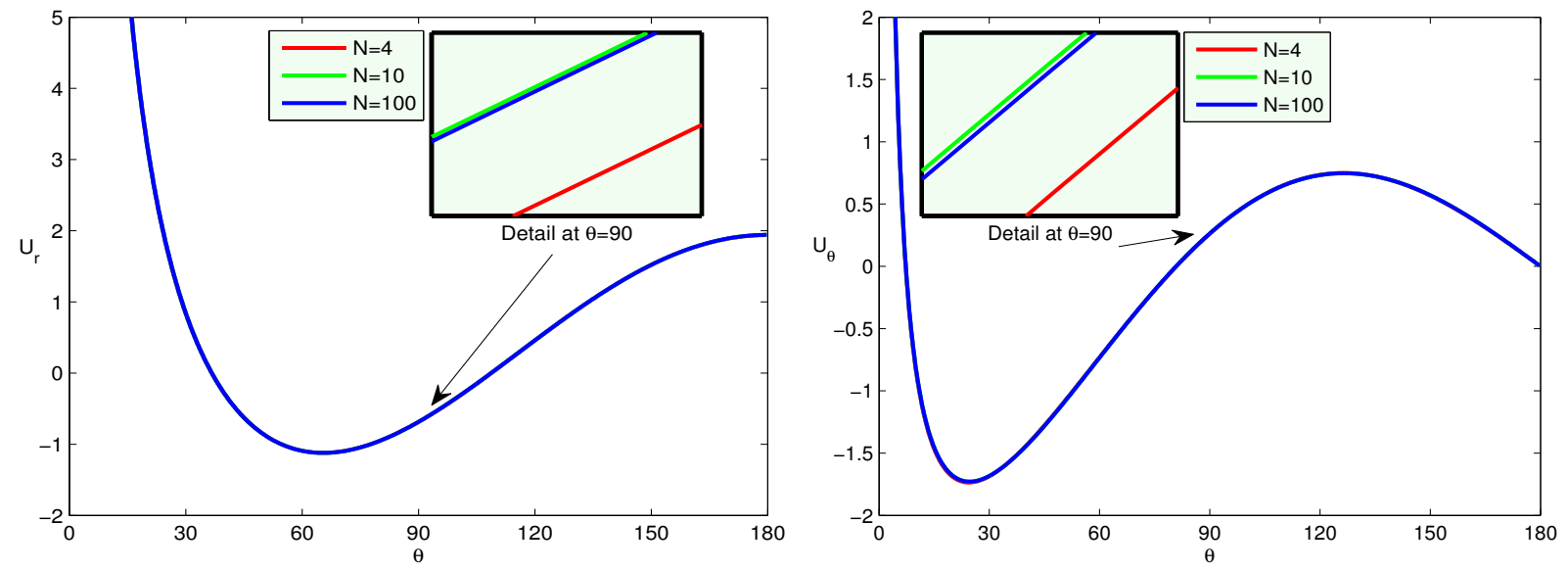

Figure 1. Convergence of the proposed expression for $U_{r}$ (left) and $U_{\theta}$ (right), which equal $(-8 \pi G R / F)$ times the functions $u_{r}$ and $u_{\theta}$ in (11). The inset on the left shows the $U_{r}$ graphs around $\theta=\pi / 2$ magnified 11250 times: the difference in the value of $U_{r}$ from $N=4$ to $N=10$ is $4.5259 \cdot 10^{-4}$, and from $N=10$ to $N=100$ it is $-1.7059 \cdot 10^{-5}$. The inset on the right shows the $U_{\theta}$ graphs around $\theta=\pi / 2$ magnified 1200 times: the difference in the value of $U_{\theta}$ from $N=4$ to $N=10$ is $2.4097 \cdot 10^{-3}$, and from $N=10$ to $N=100$ it is $-1.5040 \cdot 10^{-4}$. 

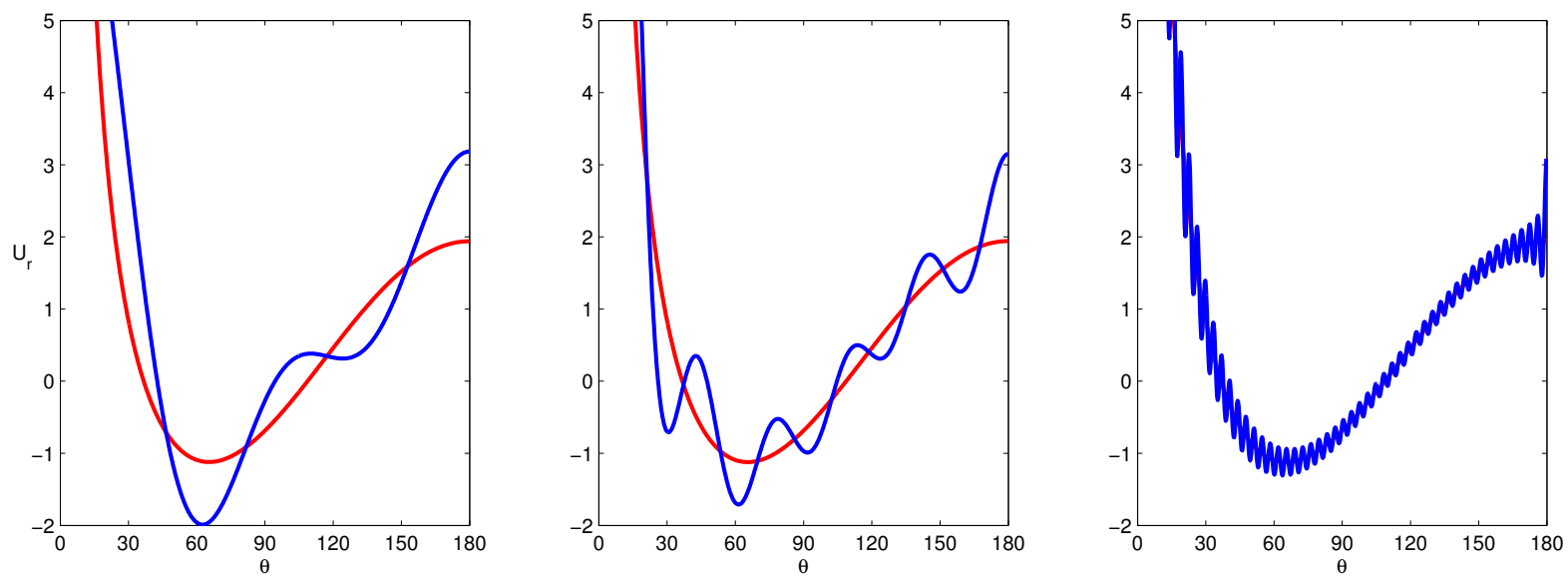

Figure 2. Comparison of the convergence of $U_{r}=(-8 \pi G R / F) u_{r}$ with the truncation value $N$ for the existing expression ((6), shown in blue) and the expression proposed herein ((11), shown in red). From left to right: $N=4,10,100$.
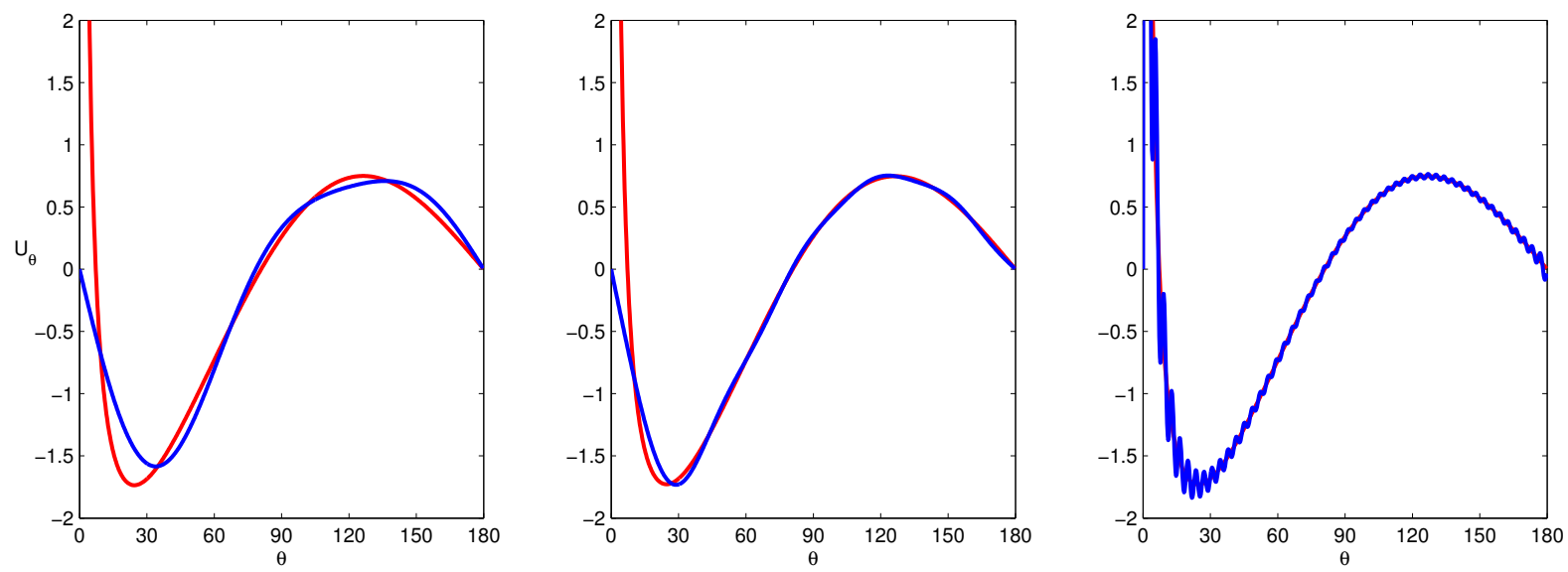

Figure 3. Comparison of the convergence of $U_{\theta}=(-8 \pi G R / F) u_{\theta}$ with the truncation value $N$ for the existing expression ((6), shown in blue) and the expression proposed herein ((11), shown in red). From left to right: $N=4,10,100$.

By design, the proposed expression, (11), converges much faster than the existing expression, (6), as seen in Figures 2 and 3. Looking at the convergence of the proposed expressions with the truncation value $N$, Figure 1, we can suggest that the analytic portion of the expression alone gives close results. However, it should be noted that one cannot get rid of the first two terms in the series for $f(\theta)$ and $g(\theta)$ because of their large magnitudes. As far as the general behavior of the normalized displacements with $\theta$, we see that they increase asymptotically approaching $\theta=0$, change sign between $36.7^{\circ}$ and $108.7^{\circ}$ for $U_{r}\left(7.27^{\circ}\right.$ and $80.83^{\circ}$ for $\left.U_{\theta}\right)$, and have a minimum at $65.5^{\circ}$ for $U_{r}\left(24.6^{\circ}\right.$ for $\left.U_{\theta}\right)$. This is difficult to see in the figures, but due to the symmetry of the loading, the displacement $U_{\theta}$ must have a value of 0 at $\theta=0$. 


\section{Green's function}

4.1. A fast convergent form for the Green's function. The surface displacements for arbitrary loading may be written, by analogy with the ansatz (8) for the point force, and generalizing the latter,

$$
\begin{aligned}
& u_{r}(R, \theta)=\frac{R}{4 G} \int_{0}^{\pi}\left(4(1-v) S(\theta, \phi)+\sum_{j=0}^{M} a_{j}(v) S_{j}(\theta, \phi)+f(\theta, \phi)\right) \sigma(\phi) \sin \phi d \phi, \\
& u_{\theta}(R, \theta)=\frac{R}{4 G} \frac{d}{d \theta} \int_{0}^{\pi}\left(\sum_{j=0}^{M} b_{j}(v) S_{j}(\theta, \phi)+g(\theta, \phi)\right) \sigma(\phi) \sin \phi d \phi,
\end{aligned}
$$

where $S(\theta, \phi)$ and $S_{j}(\theta, \phi)(j=1, \ldots M)$ are

$$
\begin{aligned}
S(\theta, \phi) & =\sum_{n=0}^{\infty} P_{n}(\cos \theta) P_{n}(\cos \phi), \\
S_{j}(\theta, \phi) & =\sum_{n=0}^{\infty} \frac{1}{n+1} P_{n+j}(\cos \theta) P_{n+j}(\cos \phi), \quad j=0,1, \ldots,
\end{aligned}
$$

and $f(\theta, \phi)$ and $g(\theta, \phi)$ are regular functions of $\theta$ defined by quickly convergent series in $n$ :

$$
f(\theta, \phi)=\sum_{n=0}^{\infty} C_{n} P_{n}(\cos \theta) P_{n}(\cos \phi), \quad g(\theta, \phi)=\sum_{n=0}^{\infty} D_{n} P_{n}(\cos \theta) P_{n}(\cos \phi) .
$$

The coefficients $a_{0}, a_{1}, \ldots, a_{M}$ are the same as before. The main complication is to find the functions (20). Thus, $S(\theta, \phi)$ follows from (52) as

$$
S(\theta, \phi)= \begin{cases}\frac{1}{\pi} \csc \frac{\theta}{2} \sec \frac{\phi}{2} K\left(\left(\cot \frac{\theta}{2} \tan \frac{\phi}{2}\right)^{2}\right), & \theta>\phi, \\ \frac{1}{\pi} \sec \frac{\theta}{2} \csc \frac{\phi}{2} K\left(\left(\tan \frac{\theta}{2} \cot \frac{\phi}{2}\right)^{2}\right), & \theta<\phi,\end{cases}
$$

where $K(m)$ is the complete elliptic integral of the first kind [Abramowitz and Stegun 1964, 17.3.1], while (50a) implies

$$
S_{01}(\theta, \phi) \equiv S_{0}(\theta, \phi)+S_{1}(\theta, \phi)= \begin{cases}-2 \log \sin \frac{\theta}{2} \cos \frac{\phi}{2}, & \theta>\phi \\ -2 \log \cos \frac{\theta}{2} \sin \frac{\phi}{2}, & \theta<\phi .\end{cases}
$$

The functions $S_{j}(\theta, \phi)$ can be determined, but their form is overly complicated, and defeats our objective of simplifying the Green's function. We therefore restrict the solution to the use of the above two series: $S(\theta, \phi)$ and $S_{01}(\theta, \phi)$. 
We therefore consider the following form of the ansatz (19) using the series $S$ and $S_{01}$ of (22) and (23), respectively. Substituting them into (19) yields the identities

$$
\begin{aligned}
u_{r}(R, \theta) & =\frac{R}{4 G} \int_{0}^{\pi}\left(4(1-v) S(\theta, \phi)+a_{01}(v) S_{01}(\theta, \phi)+f(\theta, \phi)\right) \sigma(\phi) \sin \phi d \phi \\
& =\frac{R}{4 G} \int_{0}^{\pi}\left[\sum_{n=2}^{\infty}\left(4(1-v)+a_{01}\left(\frac{2 n+1}{n(n+1)}\right)+C_{n}\right) P_{n}(\cos \theta) P_{n}(\cos \phi)\right. \\
+ & 4(1-v)\left(P_{0}(\cos \theta) P_{0}(\cos \phi)+P_{1}(\cos \theta) P_{1}(\cos \phi)\right)+a_{01}\left(P_{0}(\cos \theta) P_{0}(\cos \phi)\right. \\
+ & \left.\left.\frac{3}{2} P_{1}(\cos \theta) P_{1}(\cos \phi)\right)+C_{0} P_{0}(\cos \theta) P_{0}(\cos \phi)+C_{1} P_{1}(\cos \theta) P_{1}(\cos \phi)\right] \sigma(\phi) \sin \phi d \phi \\
u_{\theta}(R, \theta)= & \frac{R}{4 G} \frac{d}{d \theta} \int_{0}^{\pi}\left(b_{01}(v) S_{01}(\theta, \phi)+g(\theta, \phi)\right) \sigma(\phi) \sin \phi d \phi \\
= & \frac{R}{4 G} \frac{d}{d \theta} \int_{0}^{\pi}\left[\sum_{n=2}^{\infty}\left(b_{01}\left(\frac{2 n+1}{n(n+1)}\right)+D_{n}\right) P_{n}(\cos \theta) P_{n}(\cos \phi)\right. \\
+b_{01}\left(P_{0}(\cos \theta) P_{0}(\cos \phi)+\frac{3}{2} P_{1}(\cos \theta) P_{1}(\cos \phi)\right) & \left.+D_{0} P_{0}(\cos \theta) P_{0}(\cos \phi)+D_{1} P_{1}(\cos \theta) P_{1}(\cos \phi)\right] \sigma(\phi) \sin \phi d \phi .
\end{aligned}
$$

Once again we define the first two coefficients of $f(\theta, \phi)$ and $g(\theta, \phi)$ as

$$
\begin{array}{ll}
C_{0}=\frac{2(1-2 v)}{1+v}-4(1-v)-a_{01}, & C_{1}=-4(1-v)-\frac{3}{2} a_{01}, \\
D_{0}=-b_{01}, & D_{1}=-\frac{3}{2} b_{01},
\end{array}
$$

which allows us to solve the following expressions for the coefficients $a_{01}$ and $b_{01}$ :

$$
\begin{aligned}
& \sum_{n=2}^{\infty}\left(\frac{2 n+1}{n-1}\right)\left(\frac{2(1-v) n^{2}+v n-1+2 v}{n^{2}+(1+2 v) n+1+v}\right) P_{n}(\cos \theta) P_{n}(\cos \phi) \\
& =\sum_{n=2}^{\infty}\left(4(1-v)+\frac{(2 n+1)}{n(n+1)} a_{01}+C_{n}\right) P_{n}(\cos \theta) P_{n}(\cos \phi) \\
& \begin{array}{r}
\sum_{n=2}^{\infty}\left(\frac{2 n+1}{n-1}\right)\left(\frac{(-1+2 v) n+2-v}{n^{2}+(1+2 v) n+1+v}\right) P_{n}(\cos \theta) P_{n}(\cos \phi) \\
=\sum_{n=2}^{\infty}\left(\frac{(2 n+1)}{n(n+1)} b_{01}+D_{n}\right) P_{n}(\cos \theta) P_{n}(\cos \phi)
\end{array}
\end{aligned}
$$

This is done by expanding (26) for large $n$ and equating same order terms yielding

$$
a_{01}=(2 v-1)^{2}, \quad b_{01}=2 v-1 .
$$

Using (27), $C_{n}$ and $D_{n}$ are found directly from (26) (see also (18)):

$$
\begin{aligned}
C_{n}=-\frac{1}{L_{n}}\left[(v-1)(4 v-1)(4 v+1) n^{3}+\left(8 v^{2}-11 v-1\right) n^{2}\right. \\
\left.+\left(-12 v^{3}+8 v^{2}+3 v-5\right) n-(v+1)(2 v-1)^{2}\right],
\end{aligned}
$$


$D_{n}=-\frac{(2 n+1)}{L_{n}}\left[(v-1)(4 v+1) n^{2}+2\left(-v^{2}+v-1\right) n-(v+1)(2 v-1)\right]$.

In summary,

$$
\begin{aligned}
& u_{i}(R, \theta)=\frac{R}{4 G} \int_{0}^{\pi} H_{i}(\theta, \phi) \sigma(\phi) \sin \phi d \phi, \quad i=r, \theta, \\
& H_{r}(\theta, \phi)=4(1-v) S(\theta, \phi)+(1-2 v)^{2} S_{01}(\theta, \phi)+\sum_{n=0}^{\infty} C_{n} P_{n}(\cos \theta) P_{n}(\cos \phi), \\
& H_{\theta}(\theta, \phi)=\frac{d}{d \theta}\left((2 v-1) S_{01}(\theta, \phi)+\sum_{n=0}^{\infty} D_{n} P_{n}(\cos \theta) P_{n}(\cos \phi)\right),
\end{aligned}
$$

where the coefficients $C_{n}$ and $D_{n}$ are given in (28). Note that $C_{n}, D_{n}=\mathrm{O}\left(n^{-2}\right)$ as $n \rightarrow \infty$, ensuring rapidly convergent series. The Green's functions of (29) are generally valid for $\theta \in[0, \pi]$. The integrands $H_{i}(\theta, \phi)$ are smooth and bounded functions of $\phi$ for $\phi \neq \theta$, which is always the case if the displacements are evaluated at points outside the region of the loading $\sigma(\phi)$. However, for points under the load, the integration of $H_{r}(\theta, \phi)$ involves a logarithmic singularity at $\phi=\theta$. A simple means of dealing with this is described next.

4.1.1. Removing the singularity under the load. The function $S(\theta, \phi)$ exhibits a logarithmic singularity by virtue of the asymptotic behavior:

$$
K(m)=\log \frac{4}{\sqrt{1-m}}+\mathrm{O}(1-m), \quad m \uparrow 1 .
$$

The integral in (24a) is evaluated by rewriting (24a) in the equivalent form

$$
\begin{array}{r}
u_{r}(R, \theta)=\frac{R}{4 G}\left\{\int_{0}^{\phi_{0}}\left[\left(a_{01}(v) S_{01}(\theta, \phi)+f(\theta, \phi)\right) \sigma(\phi)+4(1-v)(S(\theta, \phi) \sigma(\phi)-\hat{S}(\theta, \phi) \sigma(\theta))\right] \sin \phi d \phi\right. \\
\left.+4(1-v) \sigma(\theta) \int_{0}^{\phi_{0}} \hat{S}(\theta, \phi) \sin \phi d \phi\right\}, \quad 0 \leq \theta \leq \phi_{0}, \quad(31)
\end{array}
$$

where the angle $\phi_{0}$ defines the domain of the loading, which is normally for contact problems, much less that $\pi$. The function $\hat{S}(\theta, \phi)$ has the same singularity as $S(\theta, \phi)$ and has a relatively simple integral. We choose

$$
\hat{S}(\theta, \phi)= \begin{cases}-\frac{1}{2 \pi} \csc \frac{\theta}{2} \sec \frac{\phi}{2} \log \left(\cos ^{2} \frac{\phi}{2}-\cos ^{2} \frac{\theta}{2}\right), & \theta>\phi, \\ -\frac{1}{2 \pi} \sec \frac{\theta}{2} \csc \frac{\phi}{2} \log \left(\sin ^{2} \frac{\phi}{2}-\sin ^{2} \frac{\theta}{2}\right), & \theta<\phi .\end{cases}
$$

The integrand of the first integral in (31) is now a smoothly varying function with no singularity, and the second integral is, explicitly, 


$$
\begin{aligned}
\int_{0}^{\phi_{0}} \hat{S}(\theta, \phi) \sin \phi d \phi & =-\frac{2}{\pi \sin (\theta / 2)} \int_{\cos (\theta / 2)}^{1} \log \left(x^{2}-\cos ^{2} \frac{\theta}{2}\right) d x \\
& -\frac{2}{\pi \cos (\theta / 2)} \int_{\sin (\theta / 2)}^{\sin \left(\phi_{0} / 2\right)} \log \left(x^{2}-\sin ^{2} \frac{\theta}{2}\right) d x \\
& =\frac{G(\cos (\theta / 2), 1)}{\sin (\theta / 2)}+\frac{G\left(\sin (\theta / 2), \sin \left(\phi_{0} / 2\right)\right)}{\cos (\theta / 2)}, \quad 0 \leq \theta \leq \phi_{0},
\end{aligned}
$$

where

$$
G(x, y)=-\frac{2}{\pi}((y-x) \log (y-x)+(y+x) \log (y+x)-2(y-x+x \log 2 x)) .
$$

In summary, the solution for $u_{r}$ with the singularity removed has the following form (see also (29a) for $H_{r}(\theta, \phi)$ and (33) for $\left.G(x, y)\right)$

$$
\begin{aligned}
u_{r}(R, \theta) & =\frac{R}{4 G}\left\{\int_{0}^{\phi_{0}}\left[H_{r}(\theta, \phi) \sigma(\phi)-\hat{H}_{r}(\theta, \phi) \sigma(\theta)\right] \sin \phi d \phi+h(\theta)\right\}, \\
\hat{H}_{r}(\theta, \phi) & =4(1-v) \hat{S}(\theta, \phi), \\
h(\theta) & =4(1-v)\left[\frac{G(\cos (\theta / 2), 1)}{\sin (\theta / 2)}+\frac{G\left(\sin (\theta / 2), \sin \left(\phi_{0} / 2\right)\right)}{\cos (\theta / 2)}\right] \sigma(\theta) .
\end{aligned}
$$

4.2. Examples of distributed loads. To check the convergence of the expressions in (24) we will consider a symmetric constant distributed load $\sigma(\phi)$ of the form

$$
\sigma(\phi)=\frac{-F}{\pi R^{2}} \frac{1}{\sin ^{2} \phi_{0}}, \quad 0 \leq \phi \leq \phi_{0},
$$

and a symmetric Hertzian-type load of the form

$$
\sigma(\phi)=\frac{-3 F}{2 \pi R^{2}} \frac{\sqrt{\sin ^{2} \phi_{0}-\sin ^{2} \phi}}{\sin ^{3} \phi_{0}}, \quad 0 \leq \phi \leq \phi_{0} .
$$

Both loads have been normalized such that their resultant forces are $-F$ for all ranges of the angle $\phi_{0}$, which is equivalent to the point force given by (7). The solution on the interval $0 \leq \theta \leq \phi_{0}$ is obtained using (35) and for $\phi_{0}<\theta \leq \pi$ we apply (29) directly.

Firstly, the convergence of the proposed solution, (35), is compared to the series solution for a Hertziantype load in Figures 4 and 5. These curves indicate that the convergence of the radial displacement $U_{r}$ in the proposed solution is substantially superior to the series solution. Figures 6 and 7 show the convergence of the displacements with the truncation limit $N$ under both types of loading. Subsequently, Figures 8 and 9 demonstrate that in the limit as $\phi_{0} \rightarrow 0$ the displacements due to the distributed loads approach those obtained for the point load. Moreover, the normalized radial displacement, $U_{r}$, is almost indistinguishable from the point load for a $\phi_{0}$ as large as ten degrees. A Poisson's ratio of $v=0.4$ has been used throughout.

We would also like to investigate how the displacement due to a Hertzian-type load compares with that from Hertzian contact theory. The dimensionless vertical displacement that we obtain by the methods 

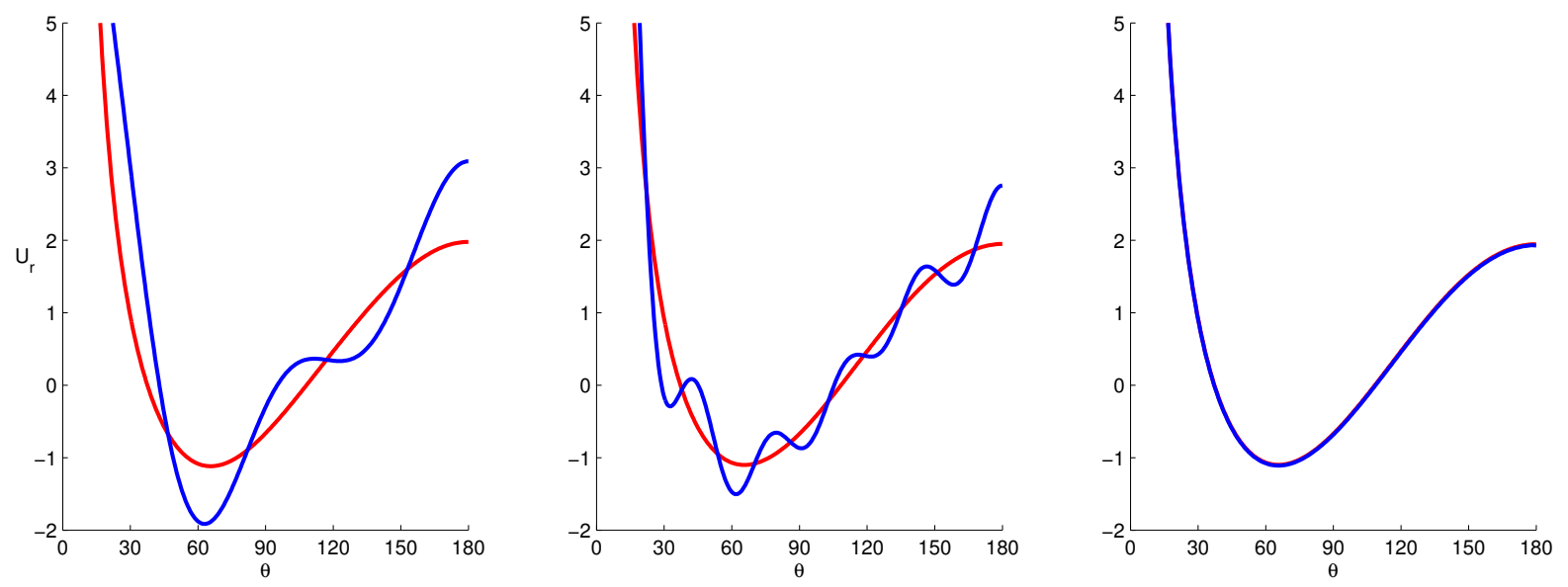

Figure 4. Comparison of the proposed solution ((35), shown in red) and existing series solutions ( $(6)$, shown in blue) for $U_{r}=(-8 \pi G R / F) u_{r}$ under a Hertzian-type load distributed up to $\phi=10^{\circ}$. From left to right: $N=4,10,100$.
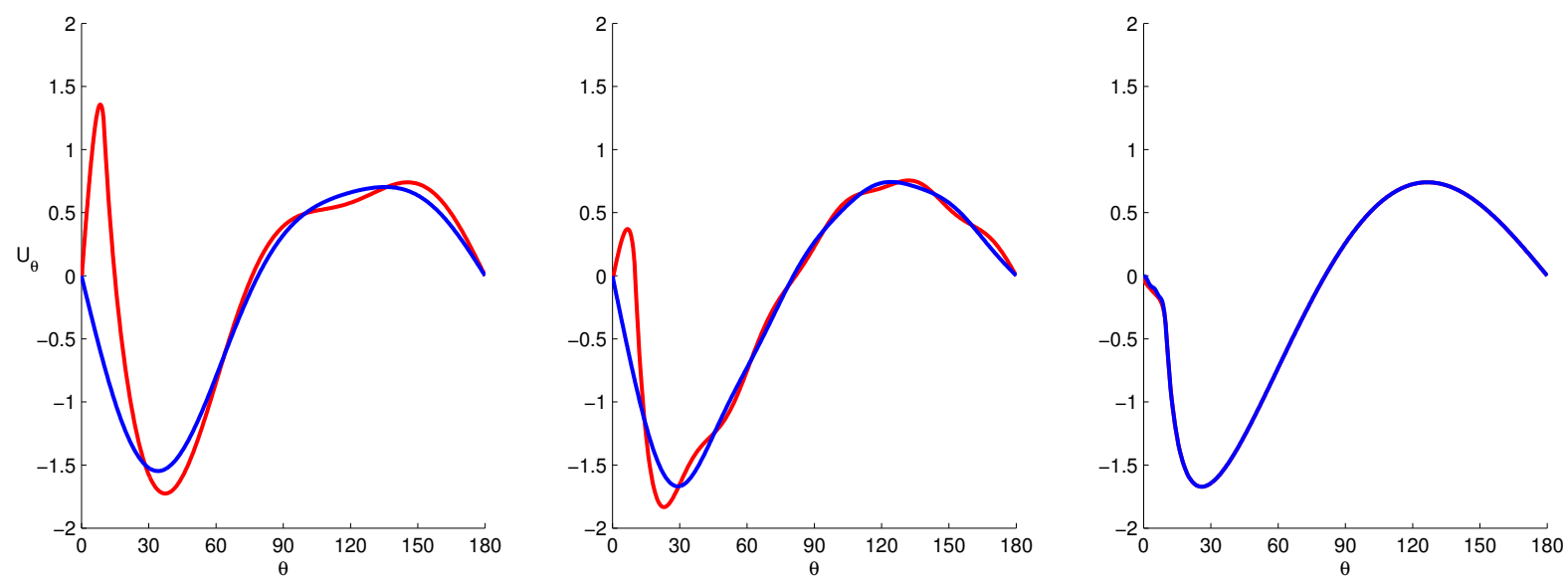

Figure 5. Comparison of the proposed solution ((35), shown in red) and existing series solutions ((6), shown in blue) for $U_{\theta}=(-8 \pi G R / F) u_{\theta}$ under a Hertzian-type load distributed up to $\phi=10^{\circ}$. From left to right: $N=4,10,100$.

outlined in this paper has the form

$$
U_{z}=U_{r} \cos \theta-U_{\theta} \sin \theta=(8 \pi G R) \frac{u_{z}}{F},
$$

where $u_{z}$ is the physical vertical displacement.

Hertz contact theory [Johnson 1985] is formulated in terms of the radius of the contact area $a$, the displacements directly under the load $\delta$, and the magnitude of the applied load $F$. We need to reformulate these quantities in terms of the contact angle $\phi_{0}$. The radius of the contact area is simply

$$
a=R \sin \phi_{0} .
$$



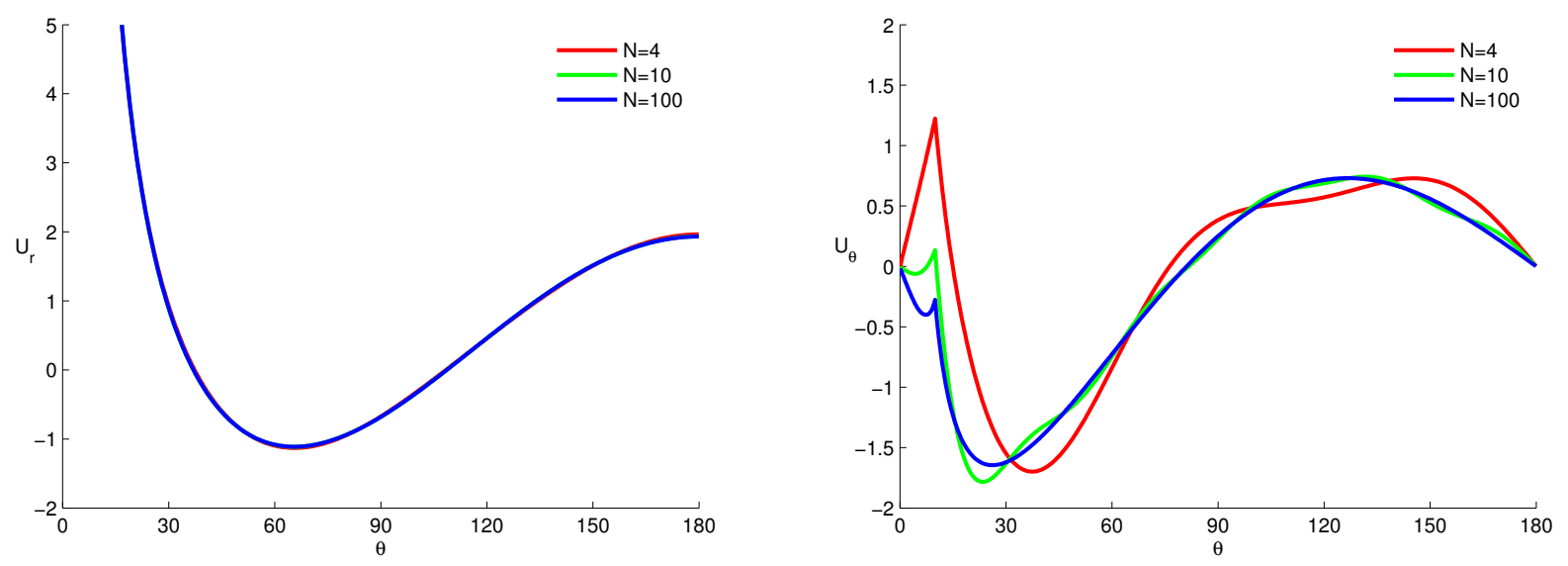

Figure 6. Convergence of the expressions for $U_{r}=(-8 \pi G R / F) u_{r}$ and $U_{\theta}=$ $(-8 \pi G R / F) u_{\theta}$ given in (24) with $N$ for a constant distributed load given by (36). The load was distributed up to $\phi_{0}=10^{\circ}$.
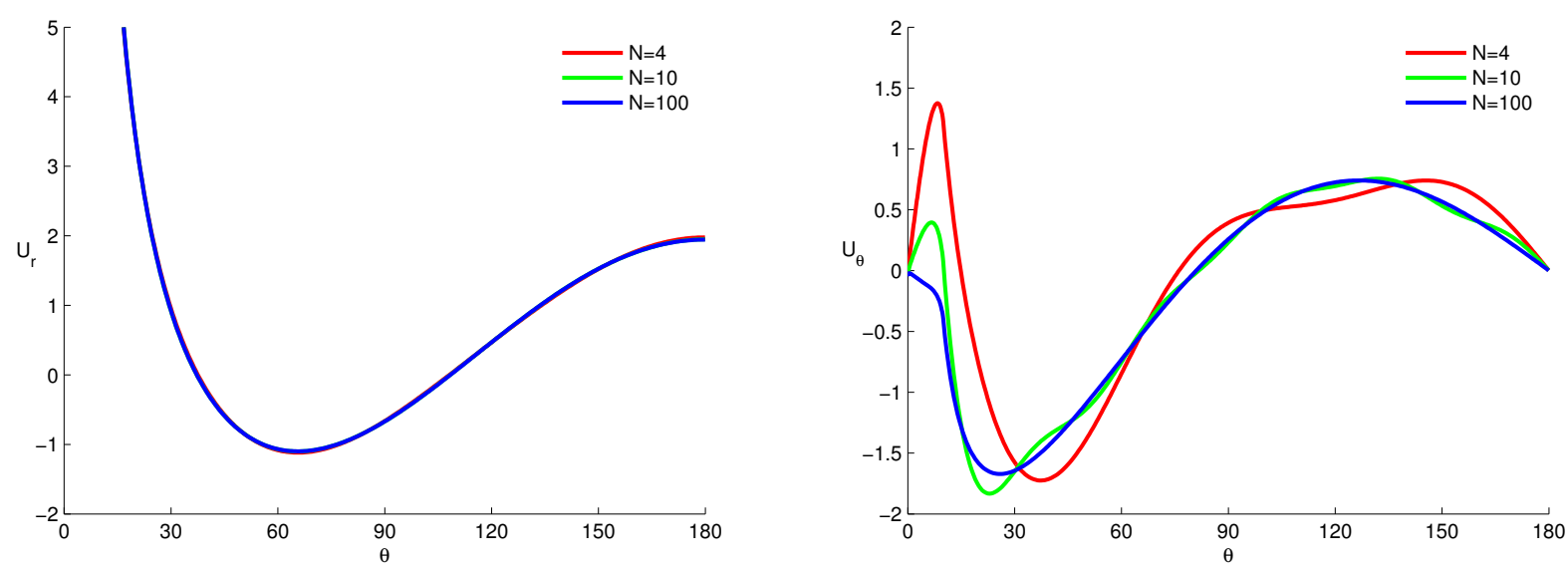

Figure 7. Convergence of the expressions for $U_{r}=(-8 \pi G R / F) u_{r}$ and $U_{\theta}=$ $(-8 \pi G R / F) u_{\theta}$ given in (24) with $N$ for a Hertzian-type distributed load given by (37). The load was distributed up to $\phi_{0}=10^{\circ}$.

The maximum vertical displacement is related to $a$ in the following manner:

$$
\delta=\frac{a^{2}}{R}=R \sin ^{2} \phi_{0}=2 u_{z}(0),
$$

where (39) was used and the last equality arises from the fact that the Hertzian solution presented here is for the contact of two spheres hence we need to halve the total displacement. Furthermore, Hertz contact theory tells us that the resultant force $F$ is proportional to $a^{3}$, or, more accurately,

$$
F=\frac{4}{3}\left(\frac{G}{1-v}\right) \frac{a^{3}}{R}=\frac{4}{3}\left(\frac{G}{1-v}\right) R^{2} \sin ^{3} \phi_{0}
$$



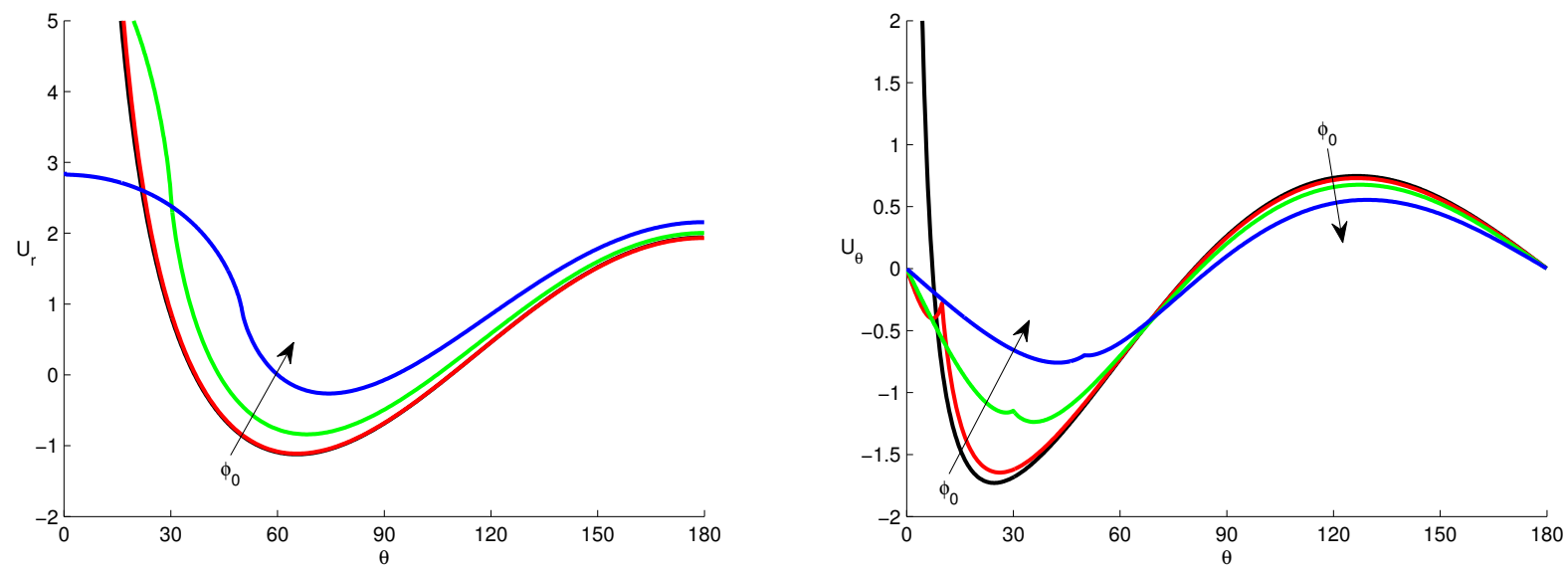

Figure 8. Comparison of the displacement due to a constant distributed load to the displacement due to a point force of the same magnitude (black). The solution was truncated at $N=300$. The loads were distributed over $\phi_{0}=10^{\circ}$ (red), 30 (green), and $50^{\circ}$ (blue).
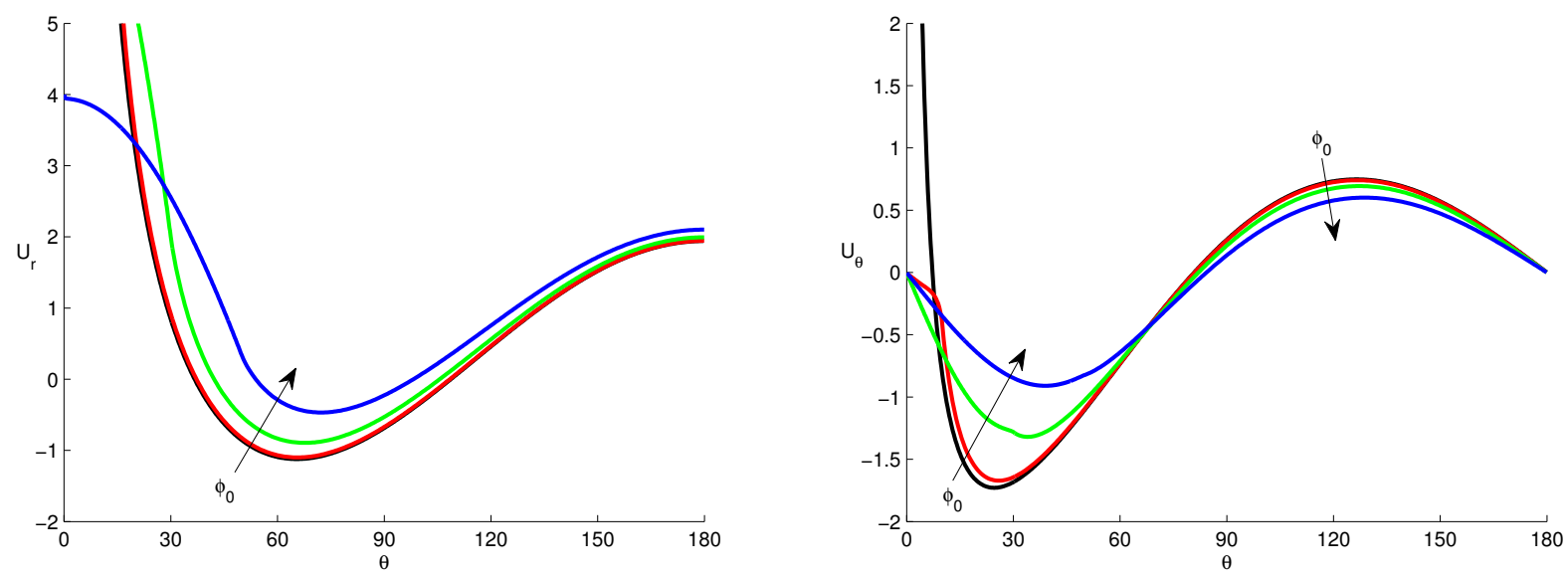

Figure 9. Convergence of the displacement due to a Hertzian-type distributed load to the displacement due to a point force of the same magnitude (black). The solution was truncated at $N=300$. The loads were distributed over $\phi_{0}=10^{\circ}$ (red), $30^{\circ}$ (green), and $50^{\circ}$ (blue).

This allows us to rewrite (38) for the dimensionless vertical displacement via Hertz contact theory, denoted as $U_{z}^{H}(0)$. Substituting (40) and (41) into (38) yields

$$
U_{z}^{H}(0)=(8 \pi G R) \frac{(R / 2) \sin ^{2} \phi_{0}}{(4 / 3)(G /(1-v)) R^{2} \sin ^{3} \phi_{0}}=\frac{3 \pi(1-v)}{\sin \phi_{0}} .
$$

Equation (42) gives us a way to compare the presented solution for the Hertzian-type load to the solution from Hertz contact theory. The numerical results are presented in Figure 10, which compares 


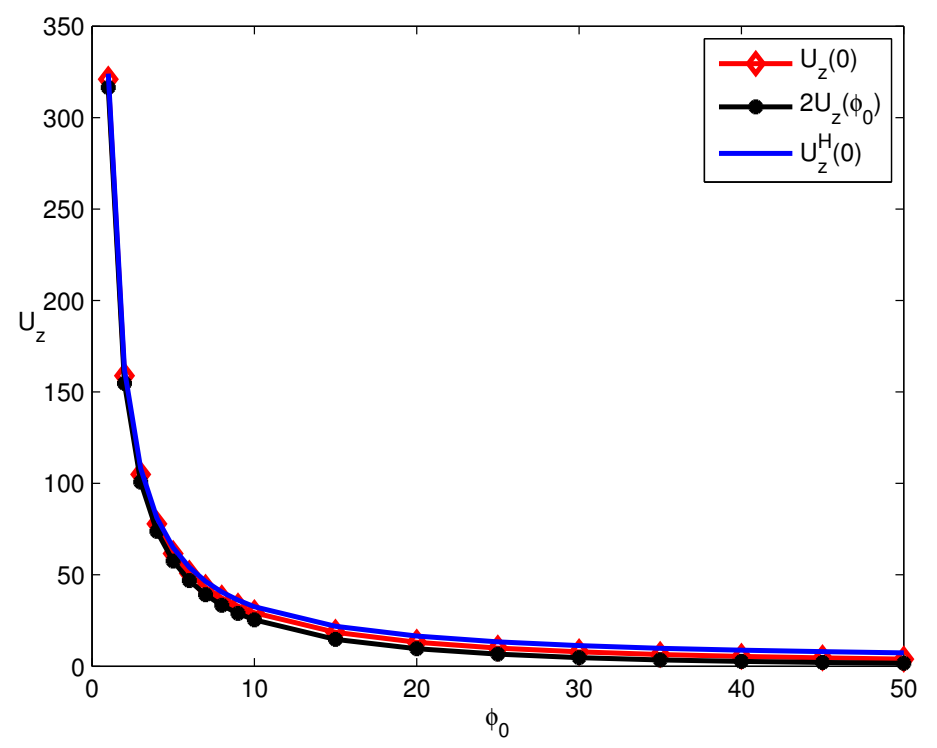

Figure 10. Comparison of the dimensionless vertical displacement $U_{z}=(8 \pi G R) u_{z} / F$ as obtained by the methods in this paper for a Hertzian-type load and that obtained from Hertz contact theory $U_{z}^{H}$ defined in (42), as a function of the contact angle $\phi_{0}$.

the vertical displacements $\left((38)\right.$ with (42)) as a function of the contact angle $\phi_{0}$. Note that along with $U_{z}(0)$ and $U_{z}^{H}(0)$ we also plot $2 U_{z}\left(\phi_{0}\right)$, which according to Hertz theory should be equal to $U_{z}(0)$. The normalized difference between the displacements is shown in Figure 11. As expected, the solutions are close for small contact areas and diverge as this area increases. The same can be said about the relationship between the displacements $U_{z}(0)$ and $2 U_{z}\left(\phi_{0}\right)$.

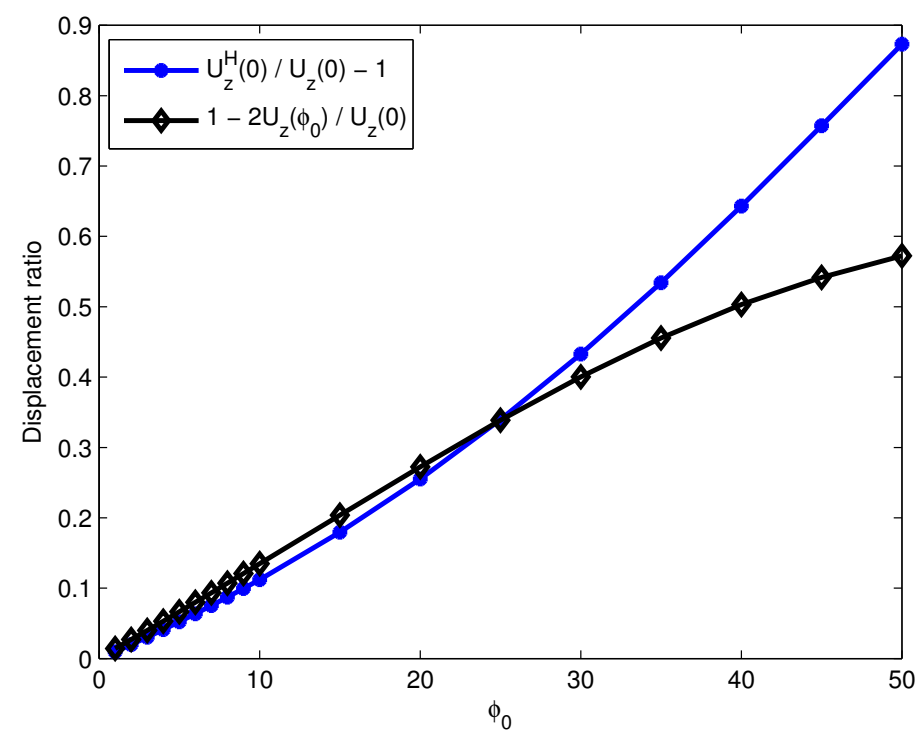

Figure 11. Normalized difference between the displacements in Figure 10 as a function of the contact angle $\phi_{0}$. 


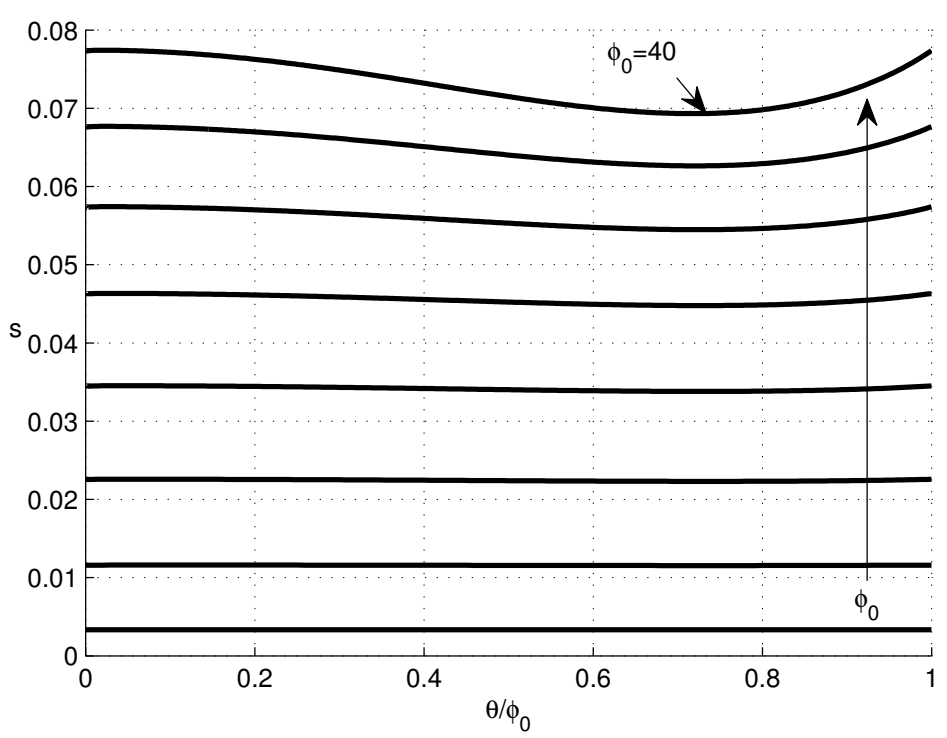

Figure 12. The flatness of the area under the load as defined in (44) as a function of the normalized angle $\theta / \phi_{0}$. Each curve corresponds to a different contact angle ranging from $\phi_{0}=5^{\circ}$ to $40^{\circ}$ in $5^{\circ}$ increments.

Comparing the maximum displacements $U_{z}(0)$ with $U_{z}^{H}(0)$ does not tell us anything about the shape of the contact area for a sphere loaded by a Hertzian-type load. Hertz contact theory states that the contact area between two identical spheres is flat, and thus we can describe it using $R\left(\cos \theta-\cos \phi_{0}\right)$. Therefore we define a function $s(\theta)$ to determine how close is our calculated displacement to the Hertzian solution as

$$
s(\theta)=k U_{z}(\theta)-\left(\cos \theta-\cos \phi_{0}\right),
$$

where $k$ is a constant determined by enforcing $s(0)=s\left(\phi_{0}\right)$, which results in

$$
s(\theta)=\frac{U_{z}(\theta)}{U_{z}(0)-U_{z}\left(\phi_{0}\right)}\left(1-\cos \phi_{0}\right)-\left(\cos \theta-\cos \phi_{0}\right) .
$$

The function $s(\theta)$ is plotted in Figure 12 for several angles $\phi_{0}$. These results show that the contact area is flat for small contact angles, but gains curvature for larger angles. According to Hertz theory, for small contact angles $\phi_{0}$, the function $s(\theta)$ behaves as a constant $s(\theta) \approx \phi_{0}^{2} / 2$. The angles shown in Figure 12 are too large to see this behavior, however, at $\phi_{0}=5^{\circ}$ the values are close with $s(\theta)=0.00334$ and $\phi_{0}^{2} / 2=0.00381$.

\section{Conclusions}

A compact Green's function for a sphere is presented which uses the fundamental idea of expressing a slowly convergent series with analytical functions and a quickly convergent series. The increased speed of convergence is demonstrated for the point force solution, which is also shown to be consistent with the more general distributed loading in the limit as the contact angle approaches zero. Since the general Green's function contains elliptical integrals, an easy method for dealing with the singularity in the 
integrand is presented. Comparing the exact displacement due to a Hertzian-type distributed load to the displacement given by Hertz contact theory we conclude that Hertz contact theory gives accurate results for contact angles up to about ten degrees, with a steadily increasing error. For larger contact angles, Hertz theory overestimates the displacements and cannot account for the shape of the contact area. This is to say that the stress distribution assumed in Hertz theory results in a curved contact surface for larger contact angles.

\section{Appendix A: Legendre polynomial formulas}

The orthogonality and completeness relations for the Legendre functions are

$$
\begin{aligned}
\frac{1}{2}(2 n+1) \int_{0}^{\pi} P_{m}(\cos \theta) P_{n}(\cos \theta) \sin \theta d \theta & =\delta_{m n}, \\
\sum_{n=0}^{\infty} \frac{1}{2}(2 n+1) P_{n}(\cos \theta) P_{n}(\cos \phi) & =\frac{\delta(\theta-\phi)}{\sin \phi},
\end{aligned}
$$

Starting with the definition for $P_{n}(x)$,

$$
P_{n}(\cos \theta)=\frac{1}{\pi} \int_{0}^{\pi}(\cos \theta+i \sin \theta \cos \alpha)^{n} d \alpha
$$

and using $\sum_{n=0}^{\infty} z^{n}=\frac{1}{1-z},|z|<1$, the well-known generating function follows:

$$
\sum_{n=0}^{\infty} t^{n} P_{n}(\cos \theta)=\frac{1}{\sqrt{1+t^{2}-2 t \cos \theta}}, \quad|t| \leq 1 .
$$

Integrating the identity (47) with respect to $t$ implies

$$
\begin{aligned}
\sum_{n=0}^{\infty} t^{n+1} \frac{P_{n}(\cos \theta)}{n+1} & =\sinh ^{-1}(\cot \theta)+\sinh ^{-1}\left(\frac{t-\cos \theta}{\sin \theta}\right) \\
& =\log \left(1+\csc \frac{\theta}{2}\right)-\sinh ^{-1}\left(\tan \frac{\theta}{2}\right)+\sinh ^{-1}\left(\frac{t-\cos \theta}{\sin \theta}\right),|t| \leq 1, \quad 0 \leq \theta \leq \pi .
\end{aligned}
$$

Taking the limit as $t \rightarrow 1$ yields (12a). $S_{1}(\theta)$ of (12b) follows from a similar result [Prudnikov et al. 1986, Equation 5.10.1.4], while $S_{2}(\theta)$ of (12c) follows from the recurrence relation

$$
(n+1) P_{n+1}(x)-(2 n+1) x P_{n}(x)+n P_{n-1}(x)=0,
$$

after dividing by $n$ and summing from $n=1$ to $\infty$ ( $S_{2}$ agrees with [Prudnikov et al. 1986, Equation 5.10.1.6]). The recurrence relation can be used to then find $S_{j}(\theta)$ for $j=3,4, \ldots$ 
A series of products of Legendre functions is given by Equations 6.11.3.1 and 6.11.3.2 of [Brychkov 2008]:

$$
\begin{aligned}
\sum_{n=1}^{\infty} \frac{2 n+1}{n(n+1)} P_{n}(x) P_{n}(y) & =-1-\log \frac{(1-x)(1+y)}{4}, \\
\sum_{n=1}^{\infty} \frac{2 n+1}{n^{2}(n+1)^{2}} P_{n}(x) P_{n}(y) & =1-\log \frac{1+y}{2} \log \frac{(1-x)(1+y)}{4}+L i_{2}\left(\frac{1+x}{2}\right)-L i_{2}\left(\frac{1+y}{2}\right),
\end{aligned}
$$

for $-1 \leq x<y \leq 1$. Equation (50a) can be derived by operating on both sides by the Legendre differential operator

$$
L_{x}=\frac{d}{d x}\left(1-x^{2}\right) \frac{d}{d x}
$$

and using the eigenvalue property $L_{x} P_{n}(x)=-n(n+1) P_{n}(x)$ to arrive at (8b) (for $x<y$ ). At the same time, the constants in the right member of (50a) follow by considering the formula for $x=0, y=1$, in which case the sum on the left can be found. Equation (50a) gives $S_{0}(\theta, \phi)+S_{1}(\theta, \phi)$ by noting that

$$
\frac{1}{n}+\frac{1}{n+1}=\frac{2 n+1}{n(n+1)}
$$

The following is a simple consequence of Legendre's addition formula [Martin 2006, Equation 3.19]:

$$
P_{n}(\cos \theta) P_{n}(\cos \phi)=\frac{1}{\pi} \int_{0}^{\pi} P_{n}(\cos \theta \cos \phi-\sin \theta \sin \phi \cos \alpha) d \alpha .
$$

Multiplying both sides of (51) by $t^{n}$ and summing implies, using (47), the identity [Prudnikov et al. 1986, Equation 5.10.2.1], for $|t|<1$,

$$
\sum_{n=0}^{\infty} t^{n} P_{n}(\cos \theta) P_{n}(\cos \phi)=\frac{4}{\pi\left(u_{+}+u_{-}\right)} K\left(\frac{u_{+}-u_{-}}{u_{+}+u_{-}}\right), u_{ \pm}=\sqrt{1-2 t \cos (\theta \pm \phi)+t^{2}}
$$

\section{Appendix B: Analytical functions and their derivatives}

We require the derivatives with respect to $\theta$ of the functions defined in (12). They are

$$
\begin{aligned}
& \frac{d S_{0}(\theta)}{d \theta}=\frac{\sin (\theta / 2)-1}{\sin \theta}, \\
& \frac{d S_{1}(\theta)}{d \theta}=-\frac{d S_{0}(\theta)}{d \theta}-\cot \frac{\theta}{2}, \\
& \frac{d S_{2}(\theta)}{d \theta}=\frac{d S_{1}(\theta)}{d \theta} \cos \theta-S_{1}(\theta) \sin \theta+\cos \frac{\theta}{2}\left(2 \sin \frac{\theta}{2}-1\right) .
\end{aligned}
$$

Similarly, the analytical function used to find $u_{\theta}$, (29), in Section 4 is

$$
S_{01}(\theta, \phi)=S_{0}(\theta, \phi)+S_{1}(\theta, \phi)= \begin{cases}-2 \log \sin \frac{\theta}{2} \cos \frac{\phi}{2}, & \theta>\phi, \\ -2 \log \cos \frac{\theta}{2} \sin \frac{\phi}{2}, & \theta<\phi .\end{cases}
$$


The derivative of $S_{01}(\theta, \phi)$ is

$$
\frac{\partial S_{01}(\theta, \phi)}{\partial \theta}=\left\{\begin{aligned}
-\cot \theta / 2, & \theta>\phi, \\
\tan \theta / 2, & \theta<\phi .
\end{aligned}\right.
$$

\section{References}

[Abramowitz and Stegun 1964] M. Abramowitz and I. A. Stegun, Handbook of mathematical functions with formulas, graphs, and mathematical tables, National Bureau of Standards Applied Mathematics Series 55, U.S. Government Printing Office, Washington, DC, 1964. Reprinted by Dover, New York, 1974.

[Bondareva 1969] V. Bondareva, "On the effect of an axisymmetric normal loading on an elastic sphere", Prikl. Mat. Mekh. 33:6 (1969), 1029-1033. In Russian; translated in J. Appl. Math. Mech. 33:6 (1969), 1001-1005.

[Bondareva 1971] V. Bondareva, "Contact problems for an elastic sphere”, Prikl. Mat. Mekh. 35:1 (1971), 61-70. In Russian; translated in J. Appl. Math. Mech. 35:1 (1971), 37-45.

[Brychkov 2008] Y. A. Brychkov, Handbook of special functions: derivatives, integrals, series and other formulas, CRC Press, Boca Raton, FL, 2008.

[Guerrero and Turteltaub 1972] I. Guerrero and M. J. Turteltaub, "The elastic sphere under arbitrary concentrated surface loads", J. Elasticity 2:1 (1972), 21-33.

[Hertz 1881] H. Hertz, "Ueber die Berührung fester elastischer Körper”, J. Reine Angew. Math. 92 (1881), 156-171.

[Johnson 1985] K. L. Johnson, Contact mechanics, Cambridge University Press, Cambridge, 1985.

[Lur'e 1955] A. I. Lur'e, Three-dimensional problems of the theory of elasticity, Gostekhizdat, Moscow, 1955.

[Martin 2006] P. A. Martin, Multiple scattering: interaction of time-harmonic waves with $N$ obstacles, Encyclopedia of Mathematics and its Applications 107, Cambridge University Press, Cambridge, 2006.

[Prudnikov et al. 1986] A. P. Prudnikov, Y. A. Brychkov, O. I. Marichev, and N. M. Queen, Integrals and series, 2: Special functions, Gordon \& Breach, New York, 1986.

[Sternberg and Rosenthal 1952] E. Sternberg and F. Rosenthal, "The elastic sphere under concentrated loads", J. Appl. Mech. (ASME) 19 (1952), 413-421.

[Villaggio 1996] P. Villaggio, "The rebound of an elastic sphere against a rigid wall", J. Appl. Mech. (ASME) 63:2 (1996), 259-263.

[Zhupanska 2011] O. I. Zhupanska, "Contact problem for elastic spheres: applicability of the Hertz theory to non-small contact areas”, Int. J. Eng. Sci. 49:7 (2011), 576-588.

Received 18 Apr 2012. Revised 4 Aug 2012. Accepted 11 Aug 2012.

AleXey S. Titovich: alexey17@eden.rutgers.edu

Department of Mechanical and Aerospace Engineering, Rutgers University, 98 Brett Road, Piscataway, NJ 08854-8058, United States

ANDREW N. NORRIS: norris@rutgers .edu

Department of Mechanical and Aerospace Engineering, Rutgers University, 98 Brett Road, Piscataway, NJ 08854-8058,

United States 


\title{
JOURNAL OF MECHANICS OF MATERIALS AND STRUCTURES
}

\author{
jomms.net
}

\author{
Founded by Charles R. Steele and Marie-Louise Steele \\ EDITORS \\ Charles R. STEele \\ DAVIDE BIGONI \\ Stanford University, USA \\ University of Trento, Italy \\ IWONA JASIUK University of Illinois at Urbana-Champaign, USA \\ YASUHIDE SHINDO Tohoku University, Japan
}

\section{EDITORIAL BOARD}

H. D. Bui École Polytechnique, France

J. P. CARTER University of Sydney, Australia

R. M. CHRISTENSEN Stanford University, USA

G. M. L. GLadWELL University of Waterloo, Canada

D. H. Hodges Georgia Institute of Technology, USA

J. Hutchinson Harvard University, USA

C. HwU National Cheng Kung University, Taiwan

B. L. KARIHALOO University of Wales, UK

Y. Y. KIM Seoul National University, Republic of Korea

Z. Mroz Academy of Science, Poland

D. PAmplona Universidade Católica do Rio de Janeiro, Brazil

M. B. RUBIN Technion, Haifa, Israel

A. N. SHUPIKOV Ukrainian Academy of Sciences, Ukraine

T. TARNAI University Budapest, Hungary

F. Y. M. WAN University of California, Irvine, USA

P. WRIGGERS Universität Hannover, Germany

W. YANG Tsinghua University, China

F. ZIEGLER Technische Universität Wien, Austria

PRODUCTION production@msp.org

SILVIO LEVY Scientific Editor

See http://jomms.net for submission guidelines.

JoMMS (ISSN 1559-3959) is published in 10 issues a year. The subscription price for 2012 is US $\$ 555 /$ year for the electronic version, and \$735/year (+\$60 shipping outside the US) for print and electronic. Subscriptions, requests for back issues, and changes of address should be sent to Mathematical Sciences Publishers, Department of Mathematics, University of California, Berkeley, CA 94720-3840.

JoMMS peer-review and production is managed by EditFLow ${ }^{\circledR}$ from Mathematical Sciences Publishers.

\author{
mathematical sciences publishers \\ http://msp.org/ \\ A NON-PROFIT CORPORATION \\ Typeset in $\mathrm{IAT}_{\mathrm{E}} \mathrm{X}$ \\ Copyright $@ 2012$ by Mathematical Sciences Publishers
}




\section{Journal of Mechanics of Materials and Structures}

\section{Volume 7, No. $7 \quad$ September 2012}

Molecular dynamics-based continuum models for the linear elasticity of nanofilms and nanowires with anisotropic surface effects

Wonbae Kim, Seung Yun Rhee and Máenghyo Cho

Characterization of human skin through skin expansion

Djenane C. Pamplona and Claudio R. Carvalho 641

In-plane dynamic excitation of AAC masonry walls patched with FRP: dynamic testing and analysis

DVIR ELMALICH and ODED RABINOVITCH

Hygrothermal analysis of exponentially graded rectangular plates

ASHRAF M. ZENKOUR 687

Green's function for symmetric loading of an elastic sphere with application to contact problems ALEXeY S. TITOVICH and ANDREW N. NORRIS 701

Multi-hit armour characterisation of metal-composite bi-layers

K. Karthikeyan, B. P. Russell, V. S. Deshpande and N. A. Fleck 